\title{
Preschoolers (Sometimes) Defer to the Majority in Making Simple Perceptual Judgments
}

\author{
Kathleen H. Corriveau and Paul L. Harris \\ Harvard University
}

\begin{abstract}
Three- and 4-year-old children were asked to judge which of a set of 3 lines was the longest, both independently and in the face of an inaccurate consensus among adult informants. Children were invariably accurate when making independent judgments but sometimes deferred to the inaccurate consensus. Nevertheless, the deference displayed by both age groups proved to be circumscribed. When asked to solve a practical problem — selecting the longest strip to build an adequate bridge — both groups relied on their own perceptual judgment, regardless of whether they had deferred to the inaccurate consensus. Confirming earlier meta-analytic findings with adults, the rate of deference was greater among Asian American children as compared with Caucasian American children.
\end{abstract}

Keywords: preschoolers, consensus, testimony, deference

When young children encounter a conflict between their own perceptual judgment and the claims made by other people, how do they respond? Do they display autonomy by maintaining their own judgment, or do they defer to the claims of other people? Recent findings suggest that preschoolers display a plausible mix of autonomy and deference. For example, Koenig and Echols (2003) studied 16-month-olds as they listened to a speaker who named a picture with either the correct or incorrect category name. If a picture of a shoe was presented, and the speaker called it a shoe, the infants generally turned to look at the named object. By contrast, if the speaker named the object incorrectly-for example, called the shoe a cup - they were likely to stare at the speaker, as if disconcerted by her error. Infants' own remarks also suggested that they had registered the error. They often named it with the correct name rather than echoing the speaker's error. A similar pattern of results was obtained with slightly older children by Pea (1982). When a speaker made a false statement-“That's a cat"with reference to a dog, both 30- and 36-month-olds often disagreed by producing an explicit denial- "No." Such denials were rare if the speaker had named the object correctly.

Not only do preschoolers generally deny claims that they judge to be false but they are also reluctant to use such claims as a basis for reasoning. For example, when presented with implausible statements- "All fish live in trees"- they are rarely willing to draw any further conclusion. If told that Tot is a fish and asked whether he lives in a tree, preschoolers typically insist that he lives

Kathleen H. Corriveau and Paul L. Harris, Human Development and Psychology, Harvard University.

This research was supported by an American Psychological Foundation Koppitz Fellowship to Kathleen H. Corriveau and by Spencer Research Grant 200700131 to Paul L. Harris. We thank the childcare centers, parents, and children who participated in this research.

Correspondence concerning this article should be addressed to Kathleen H. Corriveau, Harvard Graduate School of Education, Harvard University, 512 Larsen Hall, Appian Way, Cambridge, MA 02138. E-mail: kcorriv@fas.harvard.edu in the water and not in a tree, and they back up their conclusion by reference to their own empirical experience (Dias \& Harris, 1990; Harris, 2000; Harris \& Leevers, 2000).

Thus, preschoolers appear to have the courage of their convictions when faced with an adult's contrary assertion. Nevertheless, there are also situations in which preschoolers are deferential. Jaswal and Markman (2007) presented 2-year-olds with hybrid images that mostly resembled one entity but had some features of another. For example, children were presented with a hybrid that mostly looked like a fish but had some bird-like properties. When shown the image and asked about its habits, 2-year-olds used perceptual appearance as a guide. Thus, faced with the hybrid that mostly resembled a fish, they claimed that it lived in the water rather than in a nest. On the other hand, if an adult presented the image and claimed that it depicted a bird, children deferred to this unexpected classification and inferred properties consistent with it, saying for example that the creature lived in a nest rather than in the water.

Such deference cannot be ascribed to limited vocabulary knowledge or inadequate knowledge of category boundaries. In a follow-up study, Jaswal (2007) found that 2-year-olds with larger vocabularies - and hence more likely to be good at recognizing vocabulary boundaries - shifted the most when the hybrid was labeled unexpectedly by an adult. Moreover, 3-year-olds displayed a similar pattern (Jaswal, 2004). Indeed, when 4-year-olds were given an indication that the speaker was not simply making a mistake- "You're not going to believe this, but this is actually a bird"- they too generally responded with the same level of deference as younger children (Jaswal, 2004).

Taken together, the results of these various studies suggest that even at the beginning of language acquisition, children make firm, perceptually based judgments, resisting the counter suggestions of adults. Yet, children are not completely autonomous. Especially if there are some clues suggesting that the informant might be right, preschoolers often defer to an unexpected categorization and draw inferences accordingly. Thus, whatever the strength of their own perceptually based convictions, children will sometimes consider, 
and acquiesce to, the conflicting assertions of other people. However, the available evidence suggests that such deference is restricted to cases in which the perceptual evidence is ambiguous. The findings reported by Jaswal and his colleagues (Jaswal, 2004, 2007; Jaswal \& Markman, 2007) are based on hybrid stimuli in which there is at least some modest degree of correspondence between the unexpected claim of an adult informant and the properties of the hybrid in questions.

Are there situations in which children will defer to the unexpected claim of another person even when there is no perceptual evidence in favor of that claim? Two studies point tentatively to that possibility. Clément, Koenig, and Harris (2004, Experiment 2) gave 3- and 4-year-olds an opportunity to look at an object and note its color before it was concealed in a box. Then, two different informants - one who had described objects correctly up to that point and one who had described them incorrectly-looked in the box, and each produced misleading claims about its color. Children were then asked for their judgment. The majority of children stated the color they had seen. However, a small minority (11\% of 3 -year-olds; $22 \%$ of 4-year-olds) agreed with the reliable speaker's claim. Almost no one ( $0 \%$ of 3-year-olds; $4 \%$ of 4 -year-olds) agreed with the unreliable speaker. Similarly, Fusaro (2006) presented 3- and 4-year-olds with plausible claims (e.g., "fish live in water") and implausible claims (e.g., "fish live in trees"). A bystander either expressed nonverbal approval or disapproval of the claims made by the experimenter. Although most children did not believe the implausible claims, a minority of them (37\%) accepted the claim if it was met with bystander approval.

The findings by Clément et al. (2004) and Fusaro (2006) suggest that preschoolers might defer to an unexpected claim-even one that is in full disagreement with their own perceptual judgmentprovided the information comes from a reliable informant or more than one informant. In this respect, preschoolers may be no different from adults. Recall the classic procedure devised by Asch (1956) in which adults were presented with apparently reliable information, namely the claims of a unanimous majority that conflicted with their own perceptual judgment. Adults deferred to that unanimous majority on approximately one third of the trials.

The two studies to be reported were designed with three goals in mind. The primary goal was to check whether preschool children who are tested in a paradigm similar to that devised by Asch (1956) also defer to a unanimous majority. Recent findings show that preschoolers are sensitive to the opinion of the majority when they learn object names (Corriveau, Fusaro, \& Harris, 2009). However, object names may be particularly sensitive to majority opinion because they are a matter of social convention. Almost no research has explored whether preschoolers also defer to the majority when making unambiguous perceptual judgments-for example, when judging which of three lines is the tallest. Adults find a unanimous consensus that conflicts with their own judgment to be disturbing because there is normally a great deal of interpersonal agreement with respect to such simple perceptual judgments. In contrast, preschoolers might be less attuned to, and less disturbed by, a majority consensus that conflicts with their own judgment. Only one study that used Asch's paradigm has included preschoolers (Walker \& Andrade, 1996). In this study, a very large percentage of children $(85 \%)$ deferred to the majority (composed of three of their classmates), raising doubt about whether children had understood the task.
The second goal was to resolve an important question about any pattern of deference that children might display. Children might endorse the consensus view temporarily-without fully subscribing to it. Alternatively, they might endorse the consensus because its judgment is presumed correct. Therefore, in Experiment 2, children were also given a pragmatic task to solve to check whether any deference they had displayed should be construed as temporary or as a stable pattern of judgment.

The final goal was to explore the implications of a finding that has emerged in follow-up studies of Asch's (1956) paradigm. As noted above, adults defer to the majority on approximately one third of trials. This initial finding of Asch has proven reliable in subsequent studies of Western adults. However, the rate of deference is significantly greater among Asian adults (Bond \& Smith, 1996). We checked whether the same group difference would be found amongst preschoolers. Its emergence in early childhood would indicate that explanations for the group difference should be sought in early patterns of child rearing and not just in the values and institutions that include adults.

\section{Experiment 1}

\section{Method}

Participants. Participants included 19 children who were 3 years of age $(M=3 ; 7$ [years;months]; range $=3 ; 0-4 ; 0 ; 10$ girls $)$ and 21 children who were 4 years of age $(M=4 ; 7$; range $=$ $4 ; 2-5 ; 1 ; 11$ girls). Children were recruited from preschools in Cambridge, Massachusetts. Although socioeconomic background information was not collected for individual participants, the preschools served families from lower to upper middle class. All children in the preschool were invited to participate. Ethnicity information was collected via parental report and with help from the preschool teachers. Twenty-five were children identified as Caucasian American, 14 as Asian American, and one child as African American.

Procedure. Children were tested in two phases. First, children participated in four pretest trials in which they viewed still frames of three lines (strips of foam board) and were asked to point to the "big line." Children's ability to judge the difference between the line lengths in the absence of a majority was measured during these pretest trials. Second, children received four test trials in which they watched a movie in which three informants were asked to point to the "big line" but actually all pointed to one of the two smaller lines. Children's preference for endorsing the consensus that directly conflicted with the perceptual evidence was measured during these trials. In addition, their explicit judgments regarding the accuracy of the three informants and a memory check were obtained immediately after these four test trials. Each phase is described in more detail below.

Pretest. Four still frames of three black lines were used. Each line was constructed out of black foam board and was $2 \mathrm{~cm}$ in width and $0.5 \mathrm{~cm}$ thick. The largest (reference) lines were 30, 25, 20 , and $15 \mathrm{~cm}$. The two smaller lines in each triplet were $10 \%$ and $20 \%$ smaller than the reference. For example, for the triplet with the $30-\mathrm{cm}$ reference, the smaller lines were $27 \mathrm{~cm}$ and $24 \mathrm{~cm}$ in length. The location of the longest line (left, right, middle) varied systematically across trials. 
To introduce the task, the experimenter pointed to the first still frame of three lines and said, "See these three lines? Can you show me the big one?" Children were invited to point to the line that they thought was the largest, and their response was recorded. This was repeated for all four trials.

Test. To make the task more child friendly, we modified Asch's (1956) paradigm by presenting the group consensus via video instead of live. Video presentation has two advantages for young children beyond ensuring the uniformity of the testing situation. First, the video could be paused and/or repeated to allow for attentional lapses that are more frequent at younger ages. Indeed, as noted earlier, the only investigation that included a preschool age group (Walker \& Andrade, 1996) yielded a high rate of conformity by preschoolers, raising doubt about whether the children had understood the live task. Second, once each child was comfortable with the single experimenter, testing could proceed promptly. There was no need to familiarize children with three strangers.

To introduce the task, an unfamiliar experimenter informed the children that they would be watching a movie, and then she would ask them some questions. The experimenter pointed to a still frame of the three informants and said the following: "Oh look- here are three girls. One girl has a green shirt, and one has a blue shirt, and one has a red shirt. These girls are first going to show us which line they think is big and then I'm going to ask you what you think. Let's watch."

A film was then shown that featured three informants wearing different, solid-colored shirts (blue, green, red) and the same sets of three lines that were used during pretest trials. Trials began with a voice-over asking, "Show me the big one." All three informants simultaneously pointed to the same smaller line. The size of the line (medium, smallest) pointed to by the informants varied across trials. Finally, a still frame of the three informants with their hands by their sides was shown.

Children received three sets of questions in test trials. The four Line Judgment questions occurred immediately after viewing each video clip. The experimenter pointed to the still frame of the three informants with their hands by their sides and said the following: "They pointed to this line. What line do you think is big?"

The three Informant Judgment questions were asked following the fourth Line Judgment question. The experimenter pointed to one of the three informants and asked, "Was the girl in the green shirt very good or not very good at saying which line was big?" The same question was posed in regards to the other two informants.

Immediately following the Informant Judgment questions, children were asked two Memory Check questions. The experimenter pointed to the final triplet of three lines and asked, "Which line did the girls say was big?" Children were invited to point to the line they thought the three informants had pointed to. Next the experimenter asked, "Which line did you say was big?" Children were asked to point to the line they had previously indicated as the big line.

\section{Results}

Line judgments. Table 1 (upper panel) shows the number of children in each age group who correctly indicated the longest line on 0-4 trials. Inspection of Table 1 shows that the majority of children (58\% of 3-year-olds; $76 \%$ of 4 -year-olds) were correct on all four trials. The mean number of correct choices by 3 -year-olds
Table 1

Number of Children Who Correctly Indicated the Longest Line on 0-4 Trials by Age Group in Experiments 1 and 2

\begin{tabular}{rllllll}
\hline & \multicolumn{5}{c}{$\begin{array}{c}\text { Number of trials in which the longest } \\
\text { line was indicated }\end{array}$} \\
\cline { 2 - 6 } Experiment & 0 & 1 & 2 & 3 & 4 & $n$ \\
\hline Experiment 1 & & & & & & \\
3-year-olds & 1 & 1 & 3 & 3 & 11 & 19 \\
4-year-olds & 3 & 1 & 0 & 1 & 16 & 21 \\
Experiment 2 & & & & & & \\
3-year-olds & 2 & 2 & 1 & 7 & 8 & 20 \\
4-year-olds & 2 & 0 & 2 & 6 & 10 & 20 \\
\hline
\end{tabular}

$(M=3.16, S D=1.21,95 \% \mathrm{CI}[2.62,3.7])$ and 4-year-olds $(M=$ $3.24, S D=1.51,95 \%$ CI $[2.59,3.89])$ exceeded chance expectation (1 out of 3): 3-year-olds, $t(18)=6.56, p<.001, d=1.51$; 4-year-olds, $t(20)=5.78, p<.001, d=1.26$. An Age $\times$ Trial Number analysis of variance (ANOVA) revealed a main effect of Trial Number, $F(3,114)=3.60, p<.05, \eta^{2}=.09$, indicating that children became more resistant to the informants' judgments on later trials. No difference was found between the two age groups, $F(1,38)=0.03$, ns.

The 13 children ( 8 children 3 years of age and 5 children 4 years of age) who made errors always chose the line that had been indicated by the adult consensus as opposed to the third remaining line. The mean number of such consensus choices $(M=2.46$, $S D=1.27,95 \%$ CI $[1.77,3.15])$ exceeded chance expectation $(1$ out of 2$), t(12)=7.01, p<.001, d=1.94$.

Table 2 (upper panel) shows the number of Caucasian American and Asian American children who correctly indicated the longest line on 0-4 trials. Inspection of Table 2 shows that the majority (80\%) of Caucasian Americans but only half $(50 \%)$ of Asian Americans were correct on all four trials. Nevertheless, the mean number of correct choices by both Caucasian $(M=3.44, S D=$ $1.26,95 \%$ CI $[2.95,3.93])$ and Asian $(M=2.86, S D=1.51,95 \%$ CI $[2.09,3.65])$ children exceeded chance expectation (1 out of 3 ): Caucasian, $t(24)=8.37, p<.001, d=1.67$; Asian, $t(13)=3.78$, $p<.002, d=1.01$. A Culture $\times$ Trial Number ANOVA revealed a main effect of Trial Number, $F(3,111)=3.25, p<.05, \eta^{2}=$ .08 , showing that children became more resistant to the informants' judgments on later trials. However, the difference between Caucasian and Asian children failed to reach significance, $F(1$, $37)=1.66, n s$.

Informant judgments. Children in each age group were scored for the number of informants (maximum $=3$ ) that they judged to be "not very good" (rather than "good") at pointing to the big line. Both age groups performed above chance (1.5) in claiming that informants were "not very good": 3 -year-olds, $M=$ $2.53, S D=0.69,95 \%$ CI $[2.22,2.84], t(18)=6.42, p<.001, d=$ 1.47; 4-year-olds, $M=2.64, S D=0.87$, 95\% CI [2.27, 3.01], $t(20)=5.37, p<.001, d=1.17$. There was no significant difference between the two age groups, $t(38)=0.10$, ns.

In addition, Caucasian American and Asian American children performed above chance: Caucasian, $M=2.64, S D=0.75,95 \%$ CI [2.35, 2.93], $t(24)=7.53, p<.001, d=1.52$; Asian, $M=$ 2.36, $S D=0.84,95 \%$ CI $[1.92,2.80], t(13)=3.81, p<.001, d=$ 
Table 2

Number of Children Who Correctly Indicated the Longest Line on 0-4 Trials by Racial Group in Experiments 1 and 2

\begin{tabular}{|c|c|c|c|c|c|c|}
\hline \multirow[b]{2}{*}{ Experiment } & \multicolumn{5}{|c|}{$\begin{array}{l}\text { Number of trials in which the } \\
\text { longest line was indicated }\end{array}$} & \multirow[b]{2}{*}{$n^{\mathrm{a}}$} \\
\hline & 0 & 1 & 2 & 3 & 4 & \\
\hline \multicolumn{7}{|l|}{ Experiment 1} \\
\hline Caucasian & 2 & 1 & 1 & 1 & 20 & 25 \\
\hline Asian American & 2 & 1 & 1 & 3 & 7 & 14 \\
\hline \multicolumn{7}{|l|}{ Experiment 2} \\
\hline Caucasian & 3 & 0 & 1 & 7 & 14 & 25 \\
\hline Asian American & 1 & 2 & 2 & 5 & 4 & 14 \\
\hline
\end{tabular}

${ }^{a}$ Note that the two African American children (one in Experiment 1, one in Experiment 2) were excluded from this analysis.

1.02. There was no significant difference between the two groups, $t(37)=1.07, n s$.

To examine whether children's tendency to assert that the three informants were not very good at identifying the big line was related to their ability to autonomously choose the longest line, we created two dichotomous variables: whether children judged all three informants appropriately (i.e., as "not very good") or made at least one misjudgment, and whether children were always correct in choosing the longest line or made at least one error. A chi-square test revealed that children who judged all three informants appropriately were more likely to be always correct in choosing the longest line, $\chi^{2}(1, N=39)=12.21, p<$ $.001, \Phi=.55$.

Memory checks. Children in each age group received a point if they correctly remembered the line to which the informants pointed and the line to which they themselves had pointed (maximum $=2$ ). Both age groups performed above chance $(0.67$ out of 2 ) in remembering these two judgments: 3 -year-olds, $M=1.74$, $S D=0.45,95 \%$ CI $[1.54,1.94], t(18)=10.27, p<.001, d=$ 1.64; 4-year-olds, $M=1.86, S D=0.36,95 \%$ CI [1.71, 2.01], $t(20)=15.17, p<.001, d=2.38$. There was no significant difference between the two age groups, $t(38)=0.94, n s$.

The eight children (five children 3 years of age and three children 4 years of age) who made errors always misremembered the informants' response (i.e., they incorrectly claimed that the informants had chosen the longest line). No child made errors in remembering their own response. A binomial test confirmed that the number of children who misremembered the informants' response only (as opposed to misremembering both the informants' and their own response or misremembering only their own response) was greater than $33 \%$ chance $(p<.001)$.

Caucasian American and Asian American children performed above chance $(0.67$ out of 2$)$ in remembering the line that they themselves had pointed to and the line that the three informants had pointed to: Caucasian, $M=1.88, S D=0.33,95 \%$ CI [1.75, 2.01], $t(24)=18.24, p<.001, d=2.66$; Asian, $M=1.71, S D=$ $0.47,95 \%$ CI $[1.46,1.96], t(13)=8.33, p<.001, d=1.51$. There was no significant difference between the two groups, $t(37)=$ $1.29, n s$

Finally, to examine whether children's ability to remember their judgments and the informants' judgments was related to their performance in judging line length, two dichotomous variables were created: whether children were all correct or made at least one error on the Memory Check trials (recall that all errors were in misremembering the informants' response) and whether children were all correct or made at least one error in judging line length. A chi-square test revealed that children who were always correct in judging line length were more likely to be always correct in remembering the informants' misjudgments, $\chi^{2}(1, N=39)=$ 12.22, $p<.001, \Phi=.55$.

\section{Discussion}

Most 3- and 4-year-olds correctly identified the longest line on all four trials. However, some children made mistakes. In all such cases, children erred by choosing the line that had been indicated by the adult majority. The overall rate of conformity ( $21 \%$ of trials among 3-year-olds; $19 \%$ of trials among 4-year-olds) was slightly lower than the overall rate reported by Asch (1956). However, it should be noted that he generally used a larger majority $(N=7)$, whereas in the present study, there were only three members of the adult consensus. In addition, the majority was physically present in Asch's study, whereas we modified Asch's setup such that the preschoolers in the current study watched the informants on video. Nevertheless, a sizeable minority of preschoolers did choose the line endorsed by the majority.

When judging the accuracy of the informants, both 3- and 4-year-olds generally claimed that the informants were "not very good" at choosing the largest line. In addition, most 3- and 4-yearolds correctly remembered both the line that they had previously pointed to and the incorrect line pointed to by the three informants. Children's' ability to correctly choose the longest line on all four test trials was related to their subsequent informant judgments and their replies to memory checks. Children who were more autonomous in their line judgments (i.e., never deferred to the consensus) were more likely to make appropriately negative judgments about the informants and to correctly remember the informants' mistaken judgments.

The results of Experiment 1 also suggest that the rate of conformity varies by group. There was a tendency for Caucasian American children to conform less than Asian American children, consistent with findings from adults (Bond \& Smith, 1996). Caution is needed in interpreting this result, however, because conventional levels of significance were not reached. We consider this issue in more detail following presentation of the results of Experiment 2 .

Experiment 2 was designed to replicate and extend the findings from Experiment 1. We tested 3- and 4-year-old children using the same procedure. In addition, however, at the end of each trial, children were asked to again choose the longest line in the context of a pragmatic goal-helping a toy rabbit to ford a river by building a bridge. If children genuinely believe that the adult consensus has identified the longest line (actually a strip of foam board), they should continue to defer to that consensus when building a bridge. On the other hand, if children only comply with the majority when making an overt and public statement, they should rely on their own perceptual judgment for their own pragmatic goals. 


\section{Experiment 2}

\section{Method}

Participants. Participants were 20 children who were 3 years of age $(M=3 ; 6$; range $=3 ; 0-4 ; 0 ; 8$ girls $)$ and 20 children who were 4 years of age $(M=4 ; 7 ;$ range $=4 ; 1-5 ; 1 ; 10$ girls). Children were recruited from preschools in Buffalo, New York. Although socioeconomic background information was not collected for individual participants, the preschools served families from lower to upper middle class. Children's ethnicity information was obtained via parental report and with help from the preschool teachers. Twenty-five children were identified as Caucasian American, 14 as Asian American, and one as African American.

Procedure. Children were tested in three phases. The first two phases were identical to Experiment 1. Children first participated in four pretest trials in which they viewed still frames of three lines and were asked to point to the "big line." Next, children received four test trials in which they watched a movie in which three informants were asked to point to the "big line," but actually all informants pointed to one of the two smaller lines. Finally, children received four bridge selection trials in which they were asked to use the longest line to create a bridge to help a bunny retrieve a high-value sticker. The details of the bridge selection trials are described below.

Two black bridge ends constructed from black foam board were placed on a piece of white foam board. The two bridge ends were $2 \mathrm{~cm}$ in width and height and were placed parallel to one another at a distance such that the longest strip of each triplet would stretch from one bridge end to the other, but the other two strips would fall short. For example, for the triplet with the $30-\mathrm{cm}$ standard line, the two bridge ends were placed $29.5 \mathrm{~cm}$ apart. Thus, the medium strip $(27 \mathrm{~cm})$ and the smallest strip $(24 \mathrm{~cm})$ would not complete the bridge. A toy bunny and some stickers were also used.

Immediately after every Line Judgment trial, the experimenter showed the child the incomplete bridge structure, the three line strips that had been used in the previous video clip, and a toy bunny that was placed on one side of the incomplete bridge. To introduce the task, the experimenter showed the child two stickers and asked the child to choose the sticker she/he liked best. The high value sticker was placed on the far side of the bridge, and the low-value sticker was placed on the near side of the bridgeclosest to the bunny. The experimenter then said the following: "This bunny can give you one sticker. Right now she is stuck on this side of the river and so can only give you this sticker. But the big line can help you make a bridge and the bunny can give you the sticker on the other side of the river. Which line is the big one to help make the bridge?" Children were invited to point to the line strip that they thought was the largest, and their response was recorded. The location of the longest line (left, right, middle) varied systematically across trials. The distance between the bridge ends was then modified for the next trial. This was repeated for all four trials.

Immediately following the Memory Check questions, children were invited to check to see how well their bridges worked. Children were asked to place their strip on the bridge to see whether it was long enough to ford the river.

\section{Results}

Line judgments. Table 1 (bottom panel) shows the number of children in each age group who correctly indicated the longest line on $0-4$ trials. Inspection of Table 1 shows that $40 \%$ of 3 -year-olds and $50 \%$ of 4 -year-olds were correct on all four trials. The mean number of correct choices by 3 -year-olds $(M=2.85, S D=1.35$, $95 \% \mathrm{CI}[2.26,3.44])$ and 4-year-olds $(M=3.10, S D=1.25,95 \%$ CI $[2.55,3.65])$ exceeded chance expectation (1 out of 3): 3 -yearolds, $t(19)=5.04, p<.001, d=1.15$; 4-year-olds, $t(19)=6.32$, $p<.001, d=1.38$. An Age $\times$ Trial Number ANOVA revealed a main effect of Trial Number, $F(3,114)=6.35, p<.001, \eta^{2}=$ .14 , indicating that children became more resistant to the informants' judgments on later trials. No difference was found between the two age groups, $F(1,38)=0.24, n s$.

The 22 children (12 children 3 years of age and 10 children 4 years of age) who made errors always chose the line that had been indicated by the adult consensus as opposed to the third remaining line. The mean number of such consensus choices $(M=1.86$, $S D=1.21,95 \%$ CI $[1.35,2.37])$ exceeded chance expectation $(1$ out of 2$), t(21)=7.24, p<.001, d=1.34$.

Table 2 (bottom panel) shows the number of Caucasian American and Asian American children who correctly indicated the longest line on $0-4$ trials. Inspection of Table 2 reveals a similar pattern to Experiment 1 . The majority (56\%) of Caucasian Americans but only a minority (29\%) of Asian Americans were correct on all four trials. Nevertheless, the mean number of correct choices by both Caucasian $(M=3.16, S D=1.31,95 \%$ CI $[2.65,3.67])$ and Asian $(M=2.64, S D=1.28,95 \%$ CI [1.97, 3.31]) children exceeded chance expectation ( 1 out of 3 ): Caucasian, $t(24)=6.97$, $p<.001, d=1.39$; Asian, $t(13)=3.85, p<.002, d=1.02$. A Culture $\times$ Trial Number ANOVA confirmed the main effect of Trial Number, $F(3,111)=6.99, p<.001, \eta^{2}=.16$, showing that children became more resistant to the informants' judgments on later trials. The difference between the Caucasian American and Asian American children failed to reach significance, $F(1,37)=$ $1.89, n s$.

In both Experiments, there was a nonsignificant trend for Caucasian American children to defer to the adult majority less than Asian American children. Given the similarity of procedure across the two studies, it was judged appropriate to combine their results to examine these trends more thoroughly. The proportion of times (with an arcsin transformation) that children appropriately selected the longest line was analyzed with a three-way ANOVA of Age $\times$ Group $\times$ Trial Number. This confirmed that the mean rate of correct, nonconformist responses was greater among Caucasian American children than among Asian American children, $F(1$, 74) $=4.66, p<.05, \eta^{2}=.05$. In addition, the main effect of Trial Number confirmed, as expected, that children made more conformist responses on earlier trials than on later trials, $F(3,222)=$ $8.90, p<.001, \eta^{2}=.11$. No other main effects or interaction were found. Figure 1 illustrates both the persistent difference between the groups and the decline in the number of conformist responses over trials. The difference between the groups is similar to that seen in adult studies with Caucasian and Asian adults (Bond \& Smith, 1996).

To further confirm that the amount of nonconformist responses was greater among the Caucasian American children than among Asian American children, we examined the proportion of children 


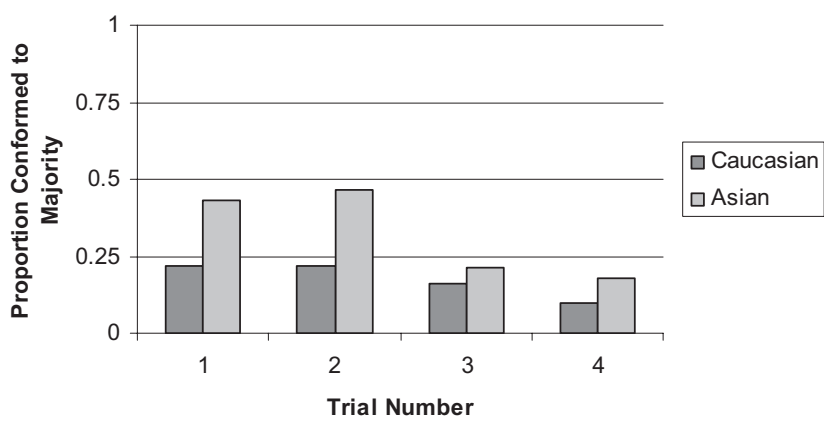

Figure 1. Proportion of children who conformed to the majority response by racial category (Caucasian American, Asian American) and trial number.

in each cultural group who were always correct in their line judgments or made at least one error across the two experiments. A chi-square analysis revealed that the number of children who were autonomous (i.e., correctly judged the length of the lines on all four trials) was greater in the Caucasian American group, $\chi^{2}(1$, $N=78)=6.06, p<.01, \Phi=.28$.

Informant judgments. Children in each age group were scored for the number of informants (maximum = 3) that they judged to be "not very good" (rather than "good") at pointing to the big line. Both age groups performed above chance (1.5) in claiming that informants were "not very good": 3-year-olds, $M=$ 2.05, $S D=1.09,95 \%$ CI $[1.57,2.53], t(19)=2.24, p<.05, d=$ 0.54 ; 4-year-olds, $M=2.65, S D=0.75$, $95 \%$ CI [2.32, 2.98], $t(19)=6.90, p<.001, d=1.53$. However, 4-year-olds performed significantly better than 3-year-olds, $t(38)=2.02, p=.05, d=$ 0.65 .

Both Caucasian American and Asian American children performed above chance (1.5) in judging that the informants were "not very good" at pointing to the big line-Caucasian, $M=2.44$, $S D=1.04,95 \%$ CI $[2.03,2.85], t(24)=4.50, p<.001, d=0.90$; Asian, $M=2.36, S D=0.89,95 \%$ CI $[1.89,2.83], t(13)=4.99$, $p<.001, d=1.33$ - and there was no significant difference between the two groups, $t(37)=0.68, n s$.

To examine whether children's tendency to assert that the three informants were not very good at identifying the big line was related to their ability to autonomously choose the longest line, we again created two dichotomous variables: whether children judged all three informants appropriately (i.e., as "not very good") or made at least one misjudgment, and whether children were always correct in choosing the longest line or made at least one error. A chi-square test revealed that children who judged all three informants appropriately were more likely to be always correct in choosing the biggest line, $\chi^{2}(1, N=39)=14.25, p<.001$, $\Phi=.59$.

Memory checks. Children in each age group received a point if they correctly remembered the line to which the informants pointed and the line to which they had pointed (maximum $=2$ ). Both age groups performed above chance ( 0.67 out of 2$)$ in remembering these judgments-3-year-olds, $M=1.35, S D=$ $0.59,95 \%$ CI $[1.09,1.61], t(19)=5.17, p<.001, d=0.59$; 4-year-olds, $M=1.85, S D=0.37,95 \%$ CI [1.69, 2.01], $t(19)=$ $14.41, p<.001, d=2.31$-and there was no significant difference between the two age groups, $t(38)=0.68, n s$.
Of the 13 children (10 children 3 years of age and 3 children 4 years of age) who made errors, 12 children misremembered the informants' response only (i.e., they incorrectly stated that the informants had chosen the longest line). Only one child misremembered his own response. A binomial test confirmed that the number of children who misremembered the informants' response only (as opposed to misremembering both the informants' and their own response or misremembering only their own response) was greater than $33 \%$ chance $(p<.001)$.

Both Caucasian American and Asian American children performed above chance ( 0.67 out of 2$)$ in remembering the line at which that they themselves had pointed and the line at which the three informants had pointed: Caucasian, $M=1.64, S D=0.59$ $95 \%$ CI $[1.40,1.87], t(24)=8.52, p<.001, d=1.12$; Asian, $M=$ $1.57, S D=0.51,95 \%$ CI $[1.30,1.84], t(13)=6.56, p<.001, d=$ 1.11. There was no significant difference between the two groups, $t(37)=0.37, n s$.

To explore whether children's ability to remember their judgments and the informants' judgments was related to their performance in judging lines, we created two dichotomous variables: whether children were all correct or made at least one error on the Memory Check trials (recall that all but one of the errors was in remembering the informants' responses), and whether children were all correct or made at least one error in judging lines. A chi-square test revealed that children who were always correct in judging lines were more likely to correctly remember the informants' response, $\chi^{2}(1, N=38)=6.01, p<.05, \Phi=.39$.

Bridge selection trials. Children received a point if they correctly chose the longest line to complete the bridge. All 3- and 4-year-olds correctly chose the longest line to complete the bridge on all four trials.

\section{Discussion}

Experiment 2 replicated key findings from Experiment 1. Children in both age groups often ignored the adult consensus and correctly identified the longest line. Nevertheless, children did sometimes make mistakes. In all such cases, they erred by choosing the line indicated by the adult consensus. The overall rate of conformity (29\% of trials among 3 -year-olds; $23 \%$ of trials among 4-year-olds) approached the overall rate reported by Asch (1956). Nevertheless, when asked to make a pragmatic decision about line length on bridge selection trials, children never deferred to the adult consensus.

As in Experiment 1, when judging the accuracy of the informants, both 3- and 4-year-olds frequently claimed that the informants were "not very good" at choosing the largest line. In addition, most 3- and 4-year-olds correctly remembered both the line that they had previously pointed to and the line pointed to by the three informants. Children who were systematically autonomous in their line judgments (i.e., never deferred to the consensus) were more likely to appropriately judge the informants as not very good at saying which line was big and more likely to identify the errors made by the informants.

Consistent with the results from Experiment 1 and with findings from adults, there was a tendency for Caucasian American children to conform less than Asian American children (Bond \& Smith, 1996). Indeed, when the results from Experiments 1 and 2 are combined, the difference between these two groups is significant. 
Asian American children were more likely to conform to the majority opinion than Caucasian American children, conforming on $40 \%$ and $18 \%$ of trials, respectively.

\section{General Discussion}

Taken together, the results of Experiments 1 and 2 support three main conclusions. First, consistent with adult research by Asch (1956) and others, children sometimes defer to a unanimous majority in making simple perceptual judgments. Second, children who do not defer are more likely to characterize the mistaken majority in a negative fashion. Third, the rate of deference to the consensus is greater among Asian American children as compared with Caucasian American children. We detail each of these conclusions in turn before considering how they might be interpreted in terms of two general response patterns: a perceptually driven pattern and a socially driven pattern.

All children accurately identified the longest line in the absence of the majority. In addition, all children were able to choose the longest line in the context of a pragmatic goal-helping a toy rabbit cross a river. Nevertheless, especially on early trials, a minority of children shifted their judgment to bring it in line with the responses made by a three-person adult majority. Such deference to a majority is consistent with the large body of findings reported by Asch (1956) and subsequent investigators (e.g., Bond \& Smith, 1996) showing that adults sometimes reject their own perception and conform to a majority judgment. Yet, the overall rate of conformity (20\% in Experiment $1,26 \%$ in Experiment 2) was slightly lower than the $33 \%$ conformity rate found in Asch's studies with adult college students. It could be that preschoolers are simply more resistant to information that is inconsistent with their own perception. Indeed, as outlined in the introduction, a growing body of research suggests that preschoolers resist information provided by adults that is inconsistent with their prior knowledge (Jaswal, 2007; Jaswal \& Markman, 2007; Koenig \& Echols, 2003; Pea, 1982).

However, in our attempt to design a child-friendly experiment, we introduced several methodological changes to the procedure adopted by Asch (1956). First, the lines themselves were larger, and the relative difference between them was slightly greater $(10 \%$ as opposed to $5 \%$ ). Second, the majority was composed of fewer informants (three informants vs. seven informants). In addition, informants were shown on video as opposed to live. Thus, preschoolers' decision to acquiesce or resist the majority did not take place in the context of a physically present majority. The latter changes would likely weaken children's tendency to conform to a majority. Nevertheless, a sizeable minority of preschoolers did conform. Accordingly, pending further research, we emphasize the qualitative parallels between the performance of adults and children rather than any subtle quantitative differences.

The extent to which children made autonomous line judgments was related to their subsequent categorizations of the informants and to their memory for the informants' line selection. More specifically, some children in each experiment were always correct in choosing the longest line. These autonomous children displayed two characteristics during postjudgment questioning. They were more likely than deferential children to consistently claim that the informants were not very good at judging the lines, and they were more likely to recall the inaccurate choices made by the majority.
In both studies, there was a nonsignificant trend for Caucasian children to defer to the adult majority less than Asian children. When results from both studies are combined, this difference is statistically significant, confirming that the mean rate of correct, nonconformist responses was greater among Caucasian American children than among Asian American children. The difference between the groups is similar to that seen in studies with Caucasian and Asian adults (Bond \& Smith, 1996).

Taken together, the results from Experiments 1 and 2 point to two different modes of responding: a perceptually driven mode and a socially driven mode. We first describe each mode and then consider their relationship particularly in relation to the group difference. Recall that children were first asked to judge the lines in the absence of the majority. They invariably made correct judgments. Even after listening to the adult consensus, children made predominantly correct judgments. Moreover, when asked to solve a practical problem, children again made correct judgments. Thus, there is ample evidence that children were fully capable of identifying the longest line independent of input from adults. This autonomous, perceptually driven mode of responding was equally evident in each age group, in both cultural groups, and in each of the two studies.

On a minority of line judgments trials, children responded in a socially driven mode. Instead of making correct judgments, they followed the adult consensus by systematically picking out the same line. Yet, it is clear that despite their deference to the adult majority, children were not persuaded that this line was actually longer. Immediately afterwards when asked to complete the bridge, children reverted to the perceptually driven mode. This disposition toward temporary agreement with the majority was associated with two other tendencies. Children who agreed with the majority were more likely to characterize the informants as good at making line judgments and more likely to forget the errors that the majority had made. In sum, responses in the socially driven mode appear to reflect a stance of "respectful deference" toward other people and toward the quality of the information that they supply but not a permanent reappraisal of the available perceptual information.

Taking these two sets of findings together, it is plausible that children faced a conflict on line judgment trials. Having examined the lines for themselves but having just heard the adult consensus, they could respond either via the perceptually driven or the socially driven mode. On most judgment trials, children resolved this conflict in favor of the perceptually driven mode. This formulation helps to pin-point the nature of the group difference. There was no evidence of any group difference in perceptually driven responses. Thus, both Caucasian and Asian children were systematically accurate in their line judgments before hearing the adult consensus and also when solving the pragmatic task. By implication, the group difference is best attributed to variation in the strength of the socially driven mode. Why might this be stronger among Asian American children?

Before considering this question, we reemphasize that the group difference was modest. It was not significant in either experiment considered in isolation. However, when the two experiments were combined, the group difference proved significant and therefore warrants discussion. We first note parallel differences between Western and East Asian adults and then consider the developmental origins of these differences. 
Research with adults has emphasized the difference between Western and East Asian systems of thought (Nisbett, Peng, Choi, \& Norenzayan, 2001). Whereas Western adults analyze situations analytically and utilize formal logic, East Asian adults are more conscious of the social context of the situation. On the basis of their heightened sensitivity to social relations, we might expect East Asian adults to be more likely to acquiesce in Asch's (1956) paradigm. Indeed, the rate of deference is significantly higher in East Asian adults than in Western adults (Bond \& Smith, 1996). Several researchers have emphasized that the different interpretations of social situations adopted by Western and East Asian adults can explain this empirical difference in the rate of deference. Although Western adults view deference as submissive conformity, East Asian adults see it as exemplifying tact and sensitivity to the context of the social relationship (Hodges \& Geyer, 2006; Packer, 2008).

Our experiments revealed a difference between Caucasian American and Asian American preschoolers. Accordingly, it is appropriate to consider the developmental origins of the differences between Western and East Asian adults highlighted above. Several studies point to deep-seated, cultural differences in the development and socialization of interpersonal relationships. First, the parenting style of Asian and Asian American mothers differs from the style used by Caucasian American mothers in early childhood (Lin \& Fu, 1990). Specifically, Asian and Asian American mothers emphasize parental control more than Caucasian American parents. This may influence their children's willingness to acquiesce to an adult consensus. Second, in a study comparing parental expectations among Japanese and American parents, Hess, Kashiwagi, Azuma, Price, and Dickson (1980) found that although there were no differences in the mean age of mastery expectations across all items, Japanese parents expected their children to master social courtesy, self-control, and compliance with adult authority earlier than their American counterparts. Similarly, Hess, Azuma, Kashiwagi, Holloway, and Wenegrat (1987) found that maternal behavior was differentially related to school readiness in Japan as compared with the United States. In Japan, the use of authority-based strategies to discipline preschoolers showed a small but positive relationship to children's later school achievement, whereas in the United States such strategies were negatively correlated with school achievement. Thus, in Japan parenting strategies that promote deference to authority proved less problematic for later cognitive achievement. Finally, Chinese preschoolers have more difficulty than American preschoolers in acknowledging when an informant is knowledgeable or ignorant (Wellman, Fang, Lui, Zhu, \& Liu, 2006). The default belief that speakers are knowledgeable may help to explain Asian American preschoolers' tendency to acquiesce to a consensus. Taken together, these various lines of research suggest that Asian and Asian American children may receive more prompting to acquiesce to other people's assertions than Caucasian American children. To test this explanation more thoroughly, it will be important for future researchers to examine larger and more homogenous samples of Asian children tested in Asian countries, such as Japan and China.

Finally, we may ask why the frequency of deference-in both cultural groups-declined across trials. A plausible answer to this question can be found in recent studies of preschoolers' selective trust in particular informants. A growing body of research shows that 3- and 4-year-olds are sensitive to informant accuracy and inaccuracy, preferring to trust an informant who has been more accurate when labeling familiar objects over 3-4 training trials (Birch, Vauthier, \& Bloom, 2008; Clément et al., 2004; Corriveau et al., 2009; Koenig, Clément, \& Harris, 2004; Koenig \& Harris, 2005; Pasquini, Corriveau, Koenig, \& Harris, 2007). Moreover, once established, this selective trust in individual informants is long-lasting. Both 3- and 4-year-olds demonstrate a preference for the accurate informant up to 1 week after their initial exposure to her (Corriveau \& Harris, 2009). Given that children build up this selective trust in individual informants across a small number of trials, it is plausible that the same mechanism can account for children's decrease in trust over four trials in the present studies. More specifically, it is plausible that children had two conflicting modes of responding on any given trial - the perceptually driven mode and the socially driven mode. Children presumably registered the conflict between these two modes, and on most trials, they concluded that the adult consensus was inaccurate. As trials proceeded, they became increasingly mistrustful of that inaccurate consensus.

In conclusion, when faced with a direct conflict between what they see and the claims of an adult majority, preschoolers usually favor their own perceptual judgment. On a minority of trials, however, they agree with the adult majority. We interpret such occasional agreement as "respectful deference." Consistent with this interpretation, it is often accompanied by positive judgments about members of the consensus, and it is more frequent among Asian American children. Nevertheless, irrespective of cultural group, children's sensitivity to the mismatch between the claims of the majority and their own perceptual judgment increasingly inhibits such deferential responding as trials proceed.

\section{References}

Asch, S. E. (1956). Studies of independence and conformity: A minority of one against a unanimous majority. Psychological Monographs, 70(9, Whole No. 416).

Birch, S. A. J., Vauthier, S. A., \& Bloom, P. (2008). Three- and 4-year-olds spontaneously use others' past performance to guide their learning. Cognition, 107, 1018-1034.

Bond, R., \& Smith, P. B. (1996). Culture and conformity: A meta-analysis of studies using Asch's (1952b, 1956) line judgment task. Psychological Bulletin, 119, 111-137.

Clément, F., Koenig, M., \& Harris, P. L. (2004). The ontogenesis of trust in testimony. Mind and Language, 19, 360-379.

Corriveau, K., Fusaro, M., \& Harris, P. L. (2009). Going with the flow: Preschoolers prefer non-dissenters as informants. Psychological Science, 20, 372-377.

Corriveau, K. H., \& Harris, P. L. (2009). Preschoolers continue to trust a more accurate informant 1 week after exposure to accuracy information. Developmental Science, 12, 188-193.

Dias, M., \& Harris, P. L. (1990). The influence of the imagination on reasoning by young children. British Journal of Developmental Psychology, 8, 305-318.

Fusaro, M. (2006). Children's use of bystanders' non-verbal cues in accepting plausible and implausible claims. Unpublished qualifying paper, Harvard University, Cambridge, MA.

Harris, P. L. (2000). The work of the imagination. Oxford, England: Blackwell.

Harris, P. L., \& Leevers, H. J. (2000). Reasoning from false premises. In P. Mitchell \& K. Riggs (Eds.), Children's reasoning and the mind (pp. 67-86). Hove, England: Psychology Press. 
Hess, R. D., Azuma, H., Kashiwagi, K., Holloway, S. D., \& Wenegrat, A. (1987). Cultural variations in socialization for school achievement: Contrasts between Japan and the United States. Journal of Applied Developmental Psychology, 8, 421-440.

Hess, R. D., Kashiwagi, K., Azuma, H., Price, G. G., \& Dickson, W. P. (1980). Maternal expectations for mastery of developmental tasks in Japan and the United States. International Journal of Psychology, 15, $259-271$.

Hodges, B. H., \& Geyer, A. L. (2006). A nonconformist account of the Asch experiments: Values, pragmatics and moral dilemmas. Personality and Social Psychology Review, 10, 2-19.

Jaswal, V. K. (2004). Don't believe everything you hear: Preschoolers' sensitivity to speaker intent in category induction. Child Development, $75,1871-1885$

Jaswal, V. K. (2007). The effect of vocabulary size on toddlers' receptiveness to unexpected testimony about category membership. Infancy, 12, $169-187$.

Jaswal, V. K., \& Markman, E. (2007). Looks aren't everything: 24-montholds willingness to accept unexpected labels. Journal of Cognition and Development, 8, 93-111.

Koenig, M., Clément, F., \& Harris, P. L. (2004). Trust in testimony: Children's use of true and false statements. Psychological Science, 10, 694-698.

Koenig, M. A., \& Echols, C. H. (2003). Infants' understanding of false labeling events: The referential roles of words and the speakers who use them. Cognition, 87, 179-208.
Koenig, M., \& Harris, P. L. (2005). Preschoolers mistrust ignorant and inaccurate speakers. Child Development, 76, 1261-1277.

Lin, C., \& Fu, V. R. (1990). A comparison of child-rearing practices among Chinese, Immigrant Chinese, and Caucasian-American parents. Child Development, 61, 429-433.

Nisbett, R. E., Peng, K., Choi, I., \& Norenzayan, A. (2001). Culture and systems of thought: Holistic versus analytic cognition. Psychological Review, 108, 291-310.

Packer, D. J. (2008). On being both with us and against us: A normative conflict model of dissent in social groups. Personality and Social Psychology Review, 12, 20-72.

Pasquini, E., Corriveau, K. H., Koenig, M., \& Harris, P. L. (2007) Preschoolers monitor the relative accuracy of informants. Developmental Psychology, 43, 1216-1226.

Pea, R. D. (1982). Origins of verbal logic: Spontaneous denials by two- and three-year-olds. Journal of Child Language, 9, 597-626.

Walker, M. B., \& Andrade, M. G. (1996). Conformity in the Asch task as a function of age. Journal of Social Psychology, 136, 367-372.

Wellman, H. M., Fang, F., Liu, D., Zhu, L., \& Liu, G. (2006). Scaling of theory-of-mind understandings in Chinese children. Psychological Science, 17, 1075-1081.

Received October 20, 2008

Revision received April 22, 2009

Accepted August 14, 2009

\section{Correction to McCartney et al. (2010)}

On the first page of the article "Testing a Series of Causal Propositions Relating Time in Child Care to Children's Externalizing Behavior," by Kathleen McCartney, Margaret Burchinal, Alison ClarkeStewart, Kristen L. Bub, Margaret T. Owen, Jay Belsky, and the NICHD Early Child Care Research Network (Developmental Psychology, 2010, Vol. 46, No. 1, pp. 1-17), author Alison ClarkeStewart's name was misspelled as Aliso Clarke-Stewart. In addition, the e-mail address listed for the corresponding author Kathleen McCartney is incorrect. The correct e-mail address is: kathleen_mccartney@gse.harvard.edu. The online versions of this article have been corrected.

DOI: $10.1037 / a 0018906$ 